\title{
Correction to: A Distributed Topic Model for Large-Scale Streaming Text
}

\author{
Yicong Li(D), Dawei Feng, Menglong Lu, and Dongsheng Li
}

\section{Correction to: \\ Chapter "A Distributed Topic Model for Large-Scale Streaming Text" in: C. Douligeris et al. (Eds.): Knowledge Science, Engineering and Management, LNAI 11776, https://doi.org/10.1007/978-3-030-29563-9_4}

The book was inadvertently published with an uncorrected version. The following corrections should have been carried out before publication:

1. Page 42: Sentence "By computing the natural gradient, corpus-level parameters are updated according to Eq. (Error! Reference source not found)-Eq. (17)." Correctly it should read "By computing the natural gradient, corpus-level parameters are updated according to Eqs. (17)-(19)."

2. Page 48: Reference [18] was inserted in the list of references but not cited in the text. It should be added in the text. The wrong sentence was on page 44 reading: "PubMed [17] is with regard to medicine papers containing 21,148,298 documents, and a vocabulary size of 196,425." Correctly it should read: "PubMed [18] is with regard to medicine papers containing $21,148,298$ documents, and a vocabulary size of 196,425."

3. Page 48: The references $[19,20]$ were inserted in the list but not cited in the text and should have been deleted. References $[21,22]$ in the reference list and the corresponding citations of them in the text have been adjusted.

The updated version of this chapter can be found at https://doi.org/10.1007/978-3-030-29563-9_4 